\title{
Making mindset matter
}

The acknowledgments for this article (BMJ 2017;356:j674, doi:10.1136/bmj.j674) were omitted. The authors thank Paul
Auerbach, Michael Ehmann, and Ted Kaptchuk for their helpful feedback on earlier drafts of this article. 